\title{
The Injury and Therapy of Reactive Oxygen Species in Intracerebral Hemorrhage Looking at Mitochondria
}

\author{
Jie Qu, Weixiang Chen, Rong Hu, and Hua Feng \\ Department of Neurosurgery, Southwest Hospital, Third Military Medical University, No. 30, Gaotanyan Street, \\ Chongqing 400038, China \\ Correspondence should be addressed to Rong Hu; huchrong@aliyun.com and Hua Feng; fenghua8888@vip.163.com
}

Received 4 December 2015; Revised 20 March 2016; Accepted 17 April 2016

Academic Editor: Qian Liu

Copyright (C) 2016 Jie Qu et al. This is an open access article distributed under the Creative Commons Attribution License, which permits unrestricted use, distribution, and reproduction in any medium, provided the original work is properly cited.

Intracerebral hemorrhage is an emerging major health problem often resulting in death or disability. Reactive oxygen species (ROS) have been identified as one of the major damaging factors in ischemic stroke. However, there is less discussion about ROS in hemorrhage stroke. Metabolic products of hemoglobin, excitatory amino acids, and inflammatory cells are all sources of ROS, and ROS harm the central nervous system through cell death and structural damage, especially disruption of the blood-brain barrier. We have considered the antioxidant system of the CNS itself and the drugs aiming to decrease ROS after ICH, and we find that mitochondria are key players in all of these aspects. Moreover, when the mitochondrial permeability transition pore opens, ROSinduced ROS release, which leads to extensive liberation of ROS and mitochondrial failure, occurs. Therefore, the mitochondrion may be a significant target for elucidating the problem of ROS in ICH; however, additional experimental support is required.

\section{Introduction}

Intracerebral hemorrhage (ICH) accounts for $9-27 \%$ of strokes worldwide. It is characterized by poor outcomes, with a high mortality rate of $30-50 \%$, and the neurological outcomes of patients who survive are also very poor $[1,2]$. The most common cause of intracerebral hemorrhage is hypertension (in approximately $65 \%$ of cases), and many other diseases, including amyloid angiopathy, brain tumours, aneurysms, arteriovenous malformations, cerebral cavernous malformations, and arteriovenous fistulae, also contribute to $\mathrm{ICH}[3]$.

Until now, there have been no effective medical or surgical therapies to improve outcomes for ICH patients. Therefore, understanding the manner in which ICH induces brain injury is important in the development of effective treatment. In addition to the initial mechanical injury produced by the hematoma, secondary injuries play an important part in further damage [3]. These secondary injuries include not only nerve cell responses to hematoma-induced stress but also the inflammatory reaction caused by the hematoma and the blood coagulation process. In the pathological process of ICH, brain cells, white matter fibre tracts, and the blood-brain barrier (BBB) are injured by the inflammatory reaction. Reactive oxygen species (ROS) are one of the most important components in the inflammatory reaction because they are both products of and participants in the reaction, causing a vicious circle.

Reactive oxygen species (ROS) are created as part of normal cellular metabolism and defence systems. Under physiological conditions, there is a balance between ROS and the antioxidant system; therefore, ROS are regulated by the antioxidant system and kept at a low level. They can take part in many cellular pathways by modulating a number of kinases, phosphatases, redox-sensitive transcription factors, and genes, which contribute to the regulation of cellular growth, differentiation, proliferation, and apoptosis [4]. However, during ICH, there are additional sources of ROS. Greater amounts of ROS can break the dynamic balance between the antioxidant system and ROS, causing cellular injury in the form of lipid peroxidation, DNA damage, and protein oxidation [5]. Therefore, ROS play an important part in the pathophysiology of ICH. ROS can initiate apoptosis and disrupt the blood-brain barrier (BBB), producing damage to the brain $[6,7]$. In this review, we will cover current research to understand $\mathrm{ICH}$-related ROS, including their 
sources, their injurious effects, their molecular mechanisms, and their relation to the antioxidant system. In addition, we will also summarize therapeutic antioxidant agents and some problems, which may contribute to the development of new therapeutic approaches.

\section{Reactive Oxygen Species}

Reactive oxygen species are highly reactive and short-lived molecules, including free radicals, such as the superoxide anion radical $\left(\mathrm{O}_{2}{ }^{\circ}\right)$ and the hydroxyl radical $\left({ }^{\circ} \mathrm{OH}\right)$, and nonradical oxidants, such as hydrogen peroxide $\left(\mathrm{H}_{2} \mathrm{O}_{2}\right)$ and singlet oxygen $\left({ }^{1} \mathrm{O}_{2}\right)$ [8]. The initial step for ROS production is the univalent reduction of molecular oxygen $\left(\mathrm{O}_{2}\right)$ to form superoxide $\mathrm{O}_{2}{ }^{\circ}$. Under normal physiological conditions, this process is mostly mediated by the mitochondrial electron transport chain (ECT) [9]. Electrons can leak from complexes $\mathrm{I}$ and III and are free to react with $\mathrm{O}_{2}$ to form the superoxide $\mathrm{O}_{2}{ }^{*}$ that is then catalysed by superoxide dismutase (SOD) to form hydrogen peroxide $\left(\mathrm{H}_{2} \mathrm{O}_{2}\right)$, which can be processed into the hydroxyl radical $\left({ }^{\circ} \mathrm{OH}\right)[10]$. The ECT, NADPH oxidase, monoaminoxidase, $\mathrm{p} 66^{\text {shc }}, \alpha$-glycerophosphate dehydrogenase, electron transfer flavoprotein (ETF) and ETF dehydrogenase, and aconitase may also contribute to the production of ROS in mitochondria [8]. Normally, most ROS can be neutralized by the antioxidant system to maintain cellular homeostasis. For example, catalase and glutathione peroxidase can convert hydrogen peroxide $\left(\mathrm{H}_{2} \mathrm{O}_{2}\right)$ to water. The "residual ROS" are also used as second messengers. They can take part in many cellular processes, such as proliferation and survival; ROS homeostasis and antioxidant gene regulation; mitochondrial oxidative stress, apoptosis, and ageing; iron homeostasis through iron-sulfur cluster proteins; and the ATM-regulated DNA damage response [4]. However, once the balance between ROS and the antioxidant system is broken by some sudden attack, such as ICH, the antioxidant system cannot eliminate the excess ROS, leading to ROS accumulation in the tissue environment. This will damage the mitochondria and lead to additional ROS release, triggering a cascade of damage in the cell.

\section{The Sources of ROS in ICH}

3.1. Hemoglobin Metabolic Products. After intracerebral hemorrhage (ICH), hematoma and perihematomal regions are rich with RBC lysis products, especially hemin (Figure 1). After intracerebral hemorrhage, red blood cells (RBC), which are present in the hematoma, lyse and release hemoglobin (Hb), which will be degraded to hemin. Hemin can be bound by hemopexin in the serum, and then the complex is transported into the cell via lipoprotein receptor-related protein (LRP1) [11]. Intracellular hemin is degraded into $\mathrm{Fe}^{2+}$, bilirubin, and carbon monoxide (CO). $\mathrm{Fe}^{2+}$ derived from hemin can generate a hydroxyl radical, which is the most reactive of all oxygen radicals, via the Fenton reaction, leading to oxidative stress $[11,12]$. Hydrocephalus after ICH is also related to iron accumulation [13]. Oxidative stress is very obvious on days 1 and 3 after ICH, but mice pretreated with deferoxamine (DFX) exhibited decreased iron accumulation and neuronal death, attenuated production of reactive oxygen species, reduced microglial activation without affecting astrocytes or neutrophil infiltration, and attenuated white matter damage [14]. Iron regulatory protein-2 (IRP2) showed effects on ferritin expression and then affected iron metabolism and neuronal vulnerability to hemoglobin. Reactive oxygen species formation and heamoxygenase-1 expression after hemoglobin treatment were also attenuated by deletion of the IRP2 gene. These results suggest that IRP2-binding activity increases the vulnerability of neurons to hemoglobin, possibly by reducing ferritin expression $[14,15]$. In addition to iron and hemoglobin, bilirubin oxidation products (BOXes) may contribute to ROS release in ICH. Clark et al. reported that production of BOXes via the hemoglobin/Fenton reaction under in vivo conditions has been seen following ICH. In hematomas from a porcine ICH model, the authors observed significant production of BOXes, malondialdehyde, and superoxide dismutase, indicating a potent oxidizing environment [16].

3.2. Excitatory Amino Acids. The initial bleed leads to an influx of glutamate from the bloodstream, and glutamate is one of the most important damaging factors in nervous system, inducing $\mathrm{Ca}^{2+}$ overload, which can lead to membrane depolarization and ROS release. Neurons are highly vulnerable to glutamate-induced excitotoxicity. Some evidence shows that glutamate can also participate in brain injury after intracerebral hemorrhage [15]. Activation of the NMDA receptor by glutamate increases $\mathrm{Ca}^{2+}$ influx, which mediates an excessive rise in cytosolic $\mathrm{Ca}^{2+}$ and consequent mitochondrial $\mathrm{Ca}^{2+}$ loading. In addition, during the pathophysiologic process of $\mathrm{ICH}$, production of thrombin after hemorrhage results in activation of Src kinase, which phosphorylates NMDA receptors, enhancing their function [17]. In addition, activation of AMPA receptors also contributes to the influx of $\mathrm{Ca}^{2+}$ and $\mathrm{Na}^{+}$, which leads to mitochondrial $\mathrm{Ca}^{2+}$ loading, and this process can be blocked by ruthenium red, a mitochondrial calcium uniporter blocker [18]. Mitochondrial $\mathrm{Ca}^{2+}$ loading contributes to the decrease in transmembrane potential and the opening of the mitochondrial permeability transition pore (MPTP), causing damage to the mitochondria and mitochondrial respiratory chain and consequent ROS release [19].

3.3. Inflammatory Cells. Microglial activation also contributes to the pathogenesis of brain injury in intracerebral hemorrhage (ICH). Tsirka has reported that inhibiting microglial activation and macrophage infiltration by the tripeptide macrophage/microglial inhibitory factor (MIF) Thr-Lys-Pro attenuated the numbers of ethidium-positive cells compared with saline-treated control mice, reduced production of reactive oxygen species, and improved neurological functional outcomes [20]. In addition, granulocytes can also be a source of ROS after ICH. They can cause the release of ROS via NADPH oxidase and myeloperoxidase [21]. Although these processes are necessary for antimicrobial defence, high ROS levels due to microglial activation and 


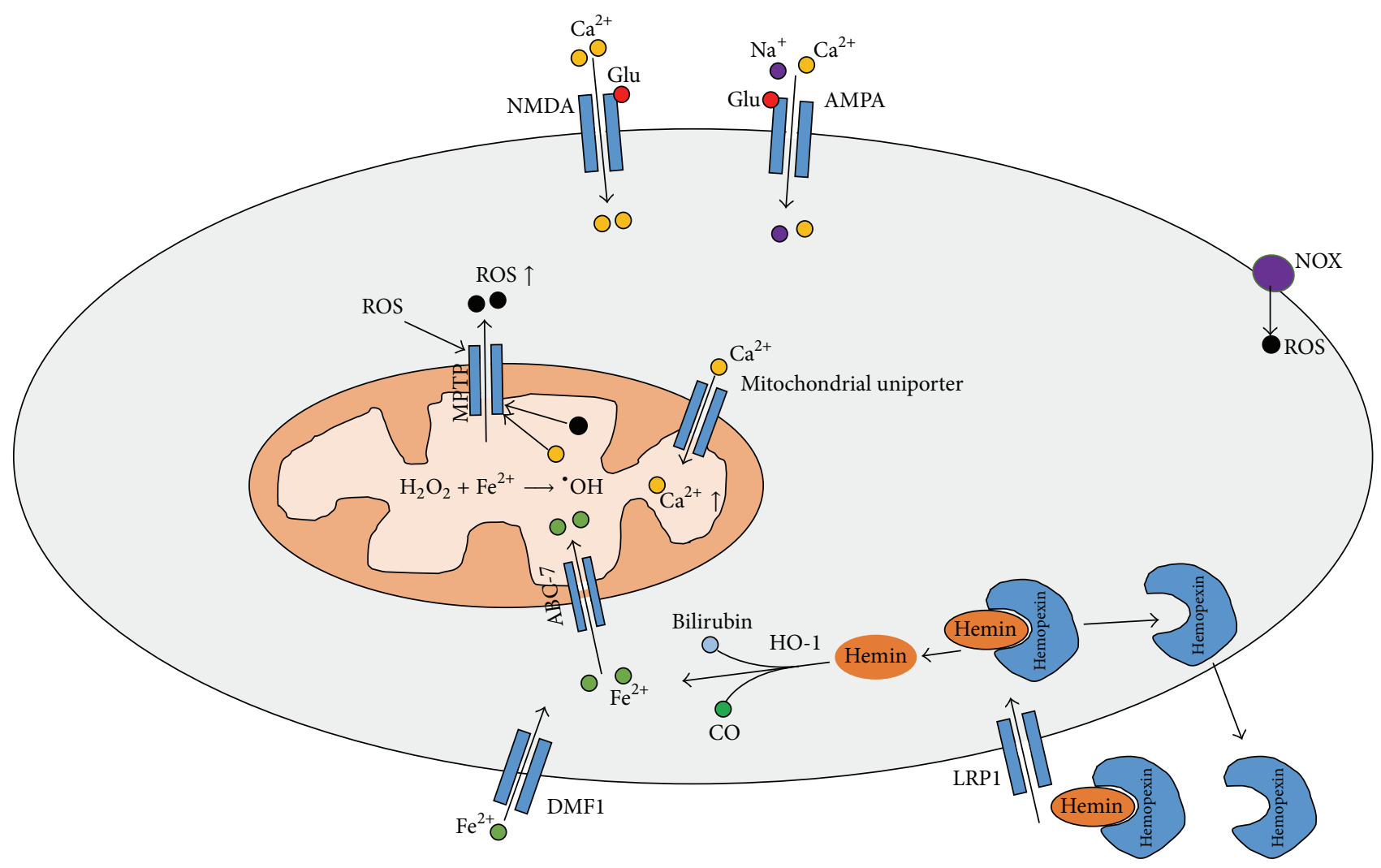

FIGURE 1: The sources of ROS in ICH: the MPTP can be opened by ROS and $\mathrm{Ca}^{2+}$, followed by ROS release. NMDA receptor activation by glutamate causes cellular $\mathrm{Ca}^{2+}$ overload, whereas APMA receptors also contribute to $\mathrm{Ca}^{2+}$ overload in the mitochondria. Ferrous iron can be transported into the cell through DMF1 and consequently loaded into the mitochondria by ABC-7; hemin binds with hemopexin and is transported into the cell through LRP1; then, inside the cell, hemin is catalysed by HO-1 into ferrous iron, which is then transported into the mitochondria; ferrous iron is used in the reaction to transform $\mathrm{H}_{2} \mathrm{O}_{2}$ into the hydroxyl radical, which is a very active radical in oxidative damage. In addition, ROS can also be produced by NOX (ROS: reactive oxygen species; $\mathrm{Fe}^{2+}$ : ferrous iron; AMPA: $\alpha$-amino-3-hydroxy5-methyl-4-isoxazole-propionic acid receptor; $\mathrm{O}_{2}{ }^{\circ}$ : superoxide radical; ${ }^{\circ} \mathrm{OH}$ : hydroxyl radical; IRP-2: iron regulatory protein-2; NMDA: N-methyl-D-aspartic acid receptor; fer-1: ferrostatin-1; DMT1: divalent metal transporter 1; HO-1: hemoxygenase-1; MPTP: mitochondrial permeability transition pore; NADPH: adenine dinucleotide phosphate; NOX: adenine dinucleotide phosphate oxidase).

neutrophil infiltration contribute to poor outcomes after ICH $[6,22]$.

3.4. ROS-Induced ROS Release. The mitochondrial permeability transition pore (MPTP) is a multiprotein complex comprising cyclophilin $\mathrm{D}$, a mitochondrial peptidylprolyl cis-trans isomerase; voltage-dependent anion channel (VDAC); adenine nucleotide translocator (ANT); and other molecule(s) that forms a channel in the mitochondrial inner membrane [23]. Its opening plays an important physiological role in maintaining healthy mitochondrial homeostasis (Figure 1). Adaptive and maladaptive responses to redox stress may involve mitochondrial channels such as the MPTP and the inner membrane anion channel (IMAC). The activation of these channels causes intracellular and intramitochondrial redox-environment changes, leading to ROS release. This regenerative cycle of mitochondrial ROS formation and release is named ROS-induced ROS release (RIRR) [8]. At higher levels of ROS, longer mPTP openings may release an ROS burst, leading to destruction of the mitochondrion and, if propagated from mitochondrion to mitochondrion, of the cell itself. Therefore, mitochondria are an important source of ROS. Following ICH, the mPTP was formed, and mROS increased, but these effects could be reversed by the VDAC inhibitor, TRO-19622, or the mROSspecific scavenger, Mito-TEMPO [24].

\section{The Contribution of ROS to Brain Injury}

4.1. Brain Cell Injury. ROS can cause cellular injury in the form of lipid peroxidation, DNA damage, and protein peroxidation, but organisms also can utilize a series of antioxidant defences, which will be discussed in next section, to protect against oxidative damage [5] (Figure 2). However, ICH-induced ROS are so abundant that antioxidant defences cannot neutralize them, leading to apoptosis through several mechanisms. The classical mechanism is caspase-dependent death: oxidative stress caused by ROS has been shown to induce cytochrome $c$ release, which is often the initiation of apoptosis [6]. ROS can permeabilize the outer mitochondrial membrane, mobilizing cytochrome $c$ from the intermembrane space into the cytosol. Released cytochrome c causes 


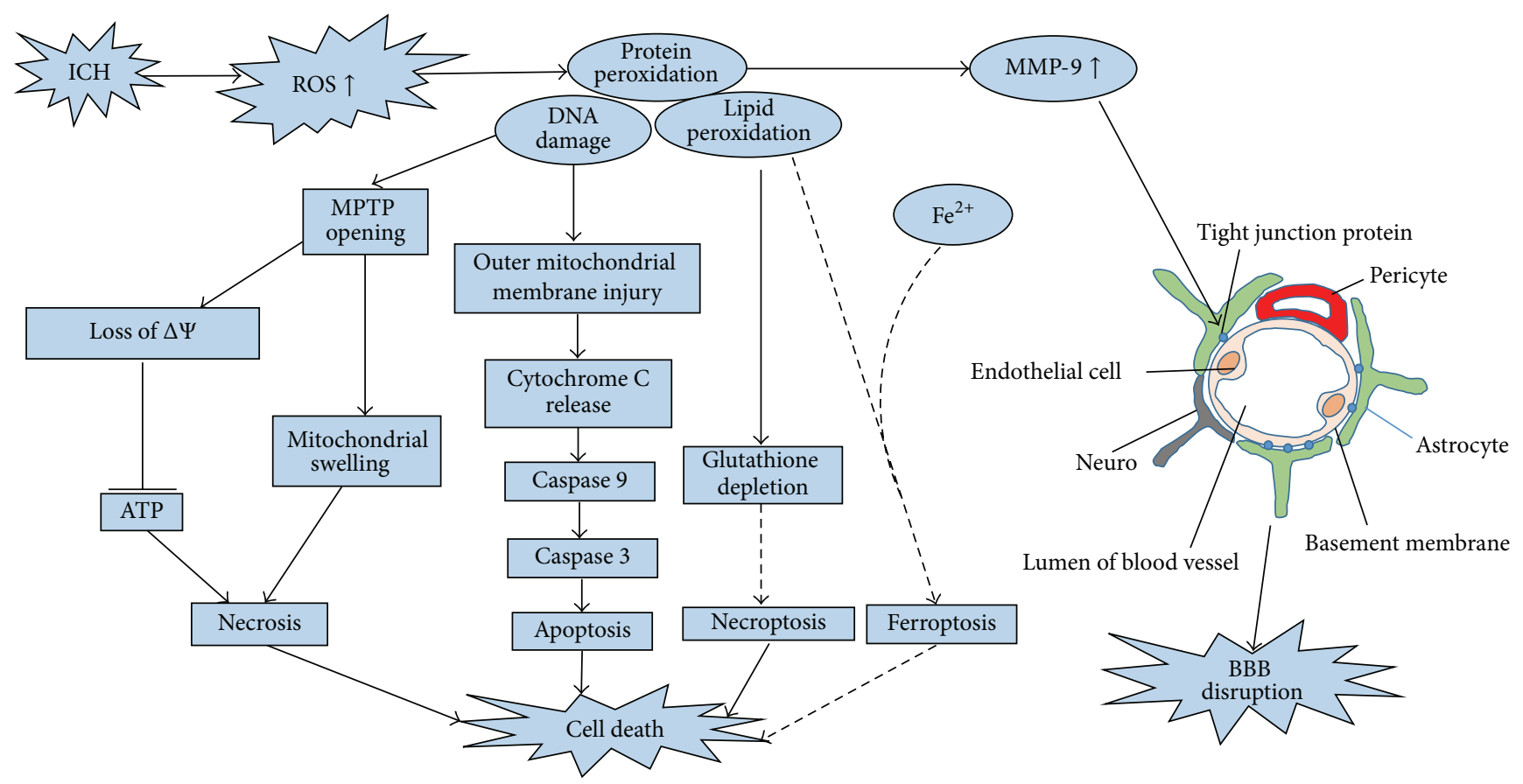

FIGURE 2: Many additional reactive oxidative species are produced after ICH. They can cause cell death and BBB disruption in the form of lipid peroxidation, DNA damage, and protein peroxidation, which contribute to the opening of the MPTP and to injury of the outer mitochondrial membrane. Once the MPTP opens, many molecules will enter into the mitochondria, and the $\Delta \Psi$ will decrease, injuring the mitochondrial respiratory chain and causing ATP depletion, causing mitochondrial swelling and necrosis. In addition, ROS can injure the outer mitochondrial membrane, causing cytochrome $\mathrm{c}$ release and activating caspase-dependent apoptosis. Excessive ROS can also cause glutathione depletion, leading to necroptosis. Ferroptosis, characterized by iron-dependent accumulation of ROS, may be a potential apoptotic mechanism during ICH. ROS also can upregulate the expression of MMP-9, degrading tight junction proteins and the basal laminar proteins, leading to BBB disruption (ICH: intracerebral hemorrhage; ROS: reactive oxygen species; BBB: blood-brain barrier; MPTP: mitochondrial permeability transition pore; $\Delta \Psi$ : transmembrane potential; MMP-9: matrix metallopeptidase 9).

formation of the apoptosome and activates initiator caspase 9, which then activates caspase 3 , leading to the inevitable onset of apoptotic death [25]. There may be other apoptotic mechanisms in addition to caspase-dependent death. In a simplified in vitro model of hemoglobin neurotoxicity, upstream and downstream caspases were upregulated, but caspase inhibition did not result in neuroprotection, and a free radical scavenger significantly reduced neuronal death, which indicated that another parallel pathway related to oxidative stress may contribute to cell death [26]. Ferroptosis, a newly recognized form of programmed cell death characterized by iron-dependent accumulation of ROS, similar to the pathological process in $\mathrm{ICH}$, may be a potential apoptosis mechanism in ICH [27]. This type of pathway is found in tumour cells, and expression of ferroptosis-related genes, such as lipocalin-2 (LCN2), a protein that participates in iron homeostasis and enhances brain iron clearance after ICH, can be tested in ICH [15]. However, existing evidence for ferroptosis is nonspecific, and more specific biomarkers are needed to characterize the pathway in ICH. Necroptosis is a type of programmed cell death that has been found in the pathological process of ICH $[28,29]$. It is characterized by defined molecular mechanisms and can be inhibited by necrostatin-1. Reactive oxygen species are also involved in the regulation of necroptosis, and glutathione depletion may play a role in necroptotic astrocyte injury after ICH [30]. The current minireview discusses the evidence for and against a role for reactive oxygen species in necroptosis. In addition, activation of the MPTP, a pore channel in the mitochondrial membrane, may also be a potential mechanism for necrosis and apoptosis. ROS produced during $\mathrm{ICH}$ can attack the mPTP. Once the mPTP opens, it will allow water, large molecules, and iron ions to enter into the mitochondrial matrix, leading to impairment of the mitochondrial respiratory chain (MRC), which results in a greater release of mitochondrial reactive oxygen species (mROS) and cell death [8]. This process has been demonstrated in ischemic stroke, but there is little research about MPTP in ICH. In an ICH mouse model, Ma et al. proved that, following ICH, MPTP is activated, causing an increase in mROS; however, the activator of the MPTP was not mentioned. Therefore, more evidence is needed to demonstrate that the MPTP can be activated by ROS after ICH.

4.2. Injury to the $B B B$. The blood-brain barrier (BBB) is a dynamic interface between the peripheral circulation and the central nervous system (CNS) that prevents toxic substances from entering into the CNS and contributes to the maintenance of brain homeostasis [31]. The BBB is formed by capillary endothelial cells, which are connected by tight 


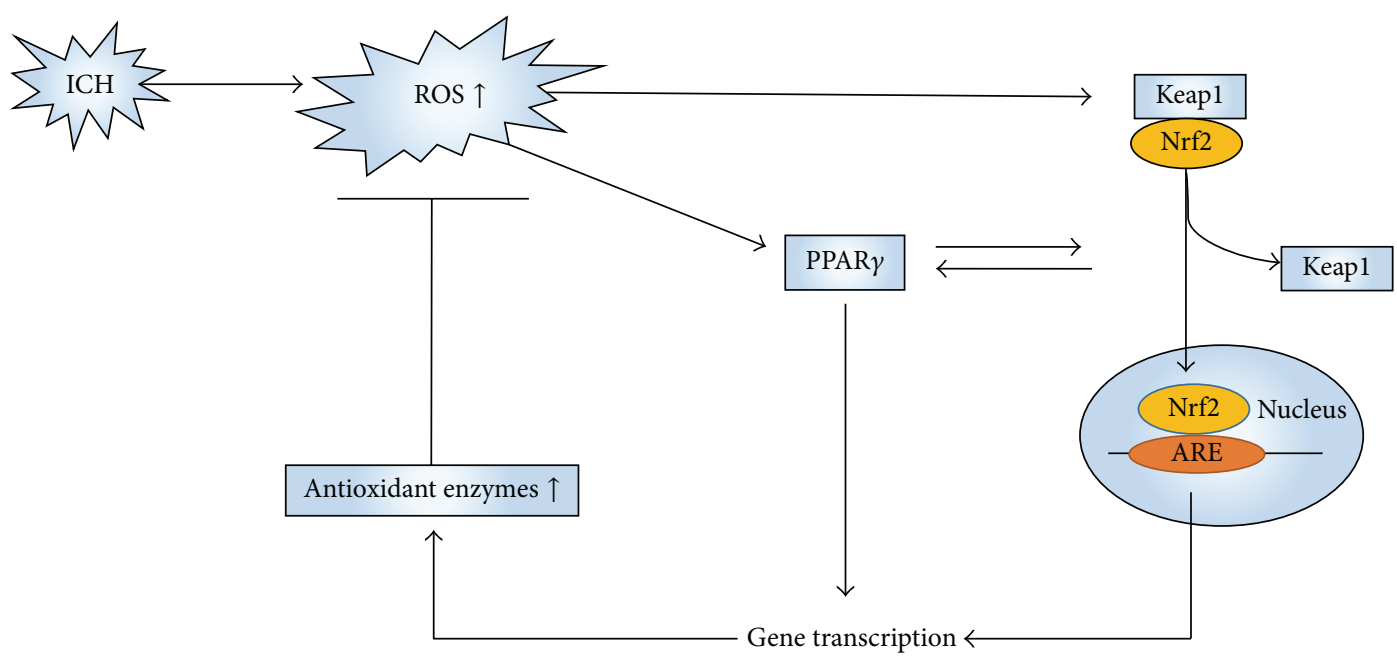

FIGURE 3: Oxidative stress can activate pathways involving Keap1 and Nrf2. Keap1 is a detector of ROS and a negative regulator of Nrf2. Under physiological conditions, Nrf2 is in a dormant state. When the brain is exposed to oxidative stress caused by ICH, Nrf2 will dissociate from Keap1, translocate to the nucleus, and activate antioxidant response element- (ARE-) dependent gene expression to neutralize ROS. Activation of PPAR $\gamma$ can lead to upregulation of the antioxidant enzymes catalase and superoxide dismutase (SOD). Nrf2: nuclear factor erythroid 2related factor; Keap1: Kelch-like ECH-associated protein 1; ICH: intracerebral hemorrhage; ROS: reactive oxygen species; PPAR $\gamma$ : peroxisome proliferator-activated receptor gamma.

junctions (TJ), together with closely associated astrocytes, pericytes, and neurons, as well as the extracellular matrix [31]. Disruption of the BBB can cause brain edema, which is an important secondary injury after ICH. Edema is related to oxidative stress (Figure 2). Matrix metalloproteinases (MMPS) comprise a family of zinc-endopeptidases that can degrade TJ proteins and basal laminar proteins, increasing the permeability of the BBB. Many researchers have demonstrated that MMPs, particularly MMP-9, are upregulated after $\mathrm{ICH}$, which is associated with oxidative stress [32]. Inhibition of oxidative stress via SOD1 overexpression also can decrease MMP-9 levels [7]. Therefore, ROS can trigger numerous molecular cascades that mediate the activation of MMPs, leading to BBB disruption.

\section{Antioxidant System in Intracerebral Hemorrhage}

To neutralize the rising ROS formed in ICH, the cellular antioxidant system is activated. The pathway involving Kelchlike ECH-associated protein 1 (Keap1) and nuclear factor erythroid 2-related factor 2 ( Nrf2) is known as the major endogenous antioxidant system [33]. It was first discovered in studies of anticarcinogenic compounds, and a beneficial role of the pathway in the progression of $\mathrm{ICH}$ has been demonstrated [34] (Figure 3). Nrf2 knockout (Nrf2 ${ }^{-/-}$) mice have greater ROS production and DNA damage than wild type (WT) mice when subjected to $24 \mathrm{~h}$ of ICH [35]. Keap1 is a detector of ROS and a negative regulator of $\mathrm{Nrf} 2$. Under physiological conditions, Nrf2 is anchored within the cytoplasm by Keap1. When the brain is exposed to oxidative stress caused by ICH, Nrf2 will dissociate from Keap1, translocate to the nucleus, and activate antioxidant response element- (ARE-) dependent gene expression [35].
Shang et al. reported that, after blood infusion in a rat model, the expression of Keap1 began to decrease at $8 \mathrm{~h}$, whereas Nrf2 began to show a significant increase at $2 \mathrm{~h}$, with a peak at $24 \mathrm{~h}$ [36]. As a result of ARE's activation, different antioxidant enzymes, including superoxide dismutase (SOD), glutathione (GSH), hemeoxygenase-1 (HO-1), glutathione-S-transferase (GST), catalase, NADPH quinone oxidoreductase-1 (NQO1), and thioredoxin (TRX), are upregulated [35]. These enzymes can partly protect the cell from the oxidative stress caused by ICH. This pathway has also become a key target for therapies in ICH because of its pleiotropic antioxidant effects. Sulforaphane, an agent that can upregulate Nrf2, reduced injury caused by ICH in mice and rats via an Nrf-dependent mechanism [37]. Chang et al. found that, in the ICH mouse model induced by injecting collagenase, (-)-epicatechin, a brain-permeable flavanol, reduced the volume of the lesion and ameliorated neurologic deficits via activation of Nrf2dependent and Nrf2-independent pathways [38]. SukumariRamesh and Alleyne reported that tert-butylhydroquinone, a selective inducer of $\mathrm{Nrf} 2$, attenuated neurodegeneration and improved neurological outcomes after ICH [39]. Furthermore, peroxisome proliferator-activated receptor gamma $(\operatorname{PPAR} \gamma)$ (Figure 3), which has pleiotropic effects regulating anti-inflammation and playing a role in glucose and lipid metabolism, appears to regulate the expression of Nrf2 $[40,41]$. Activation of PPAR $\gamma$ also may directly lead to upregulation of antioxidant enzymes, such as catalase and superoxide dismutase $[42,43]$. Therefore, $\operatorname{PPAR} \gamma$ may be another endogenous antioxidant system.

\section{Clinical Significance of ROS in ICH}

6.1. Biomarkers in ICH. To understand the pathophysiology of oxidative stress in intracerebral hemorrhage and to identify 
patient outcomes, we should take measures to evaluate oxidative stress. However, direct measurement of ROS in the brain is still difficult in humans. Therefore, several biological substances relevant to oxidative stress have been investigated as potential peripheral markers. In ischemic stroke, much research related to these biomarkers has demonstrated that biomarkers can be divided into two groups: (1) biomolecules damaged by ROS, including malondialdehyde (MDA); oxidized low-density lipoproteins (oxLDL); 8-isoprostaglandinF-2 (8-iso-PGF2), a biomarker of lipid peroxidation; and 8-hydroxy-2-deoxy-guanosine (8-OHdG), a biomarker of DNA oxidation; (2) enzymes and molecules related to the antioxidant defence system, including superoxide dismutase (SOD), glutathione peroxidase (GPX), thioredoxin (Trx), and gamma-glutamyltransferase (GGT); vitamins A (retinol), C (ascorbic acid, AA), and E; and carotenoids [44]. However, during the pathological process of $\mathrm{ICH}$, not all of the biomarkers mentioned in this passage can be detected. In a clinical study including 178 individuals (64 patients, 114 controls), ICH was significantly associated with an increased level of 8-OHdG, decreased GPX activity, and a decreased level of vitamin E, whereas MDA and vitamin A levels were not associated with ICH risk, and leukocyte 8-OhdG was better than traditional factors in predicting $\mathrm{ICH}$ outcome [45]. Uric acid (UA), an antioxidant molecule in human plasma, provides effective protection against oxidative stress in models of stroke. However, the relationship between uric acid and ICH prognosis is controversial; some authors have found that high levels of UA were correlated with a poor prognosis in ICH [46], whereas others did not conclude that uric acid levels were correlated with outcomes in ICH patients [47]. Vitamin C (ascorbic acid, AA) levels were also important indicators in ICH patients. AA levels were significantly inversely correlated with the severity of neurological impairment, as assessed by the Glasgow Coma Scale and the National Institutes of Health Stroke Scale, and with the major diameter of the lesion [48]. In conclusion, biomarkers of oxidative stress in ICH are similar to those of ischemic stroke, but there are differences between ICH and ischemic stroke, and assessment of multiple biomarkers may provide a better view of oxidative stress and outcomes in ICH patients.

6.2. Antioxidant Therapy in ICH. Therapeutic agents can be divided into two groups. One group includes agents that can prevent the formation of free radicals. Iron is a reactant in the formation of the hydroxyl radical, a highly reactive ROS, via the Fenton reaction, and iron chelating agents, such as clioquinol (CQ) and deferoxamine, reduced $\mathrm{ICH}$-induced brain edema, neuronal death, and brain atrophy in a rat ICH model [49-51]. Apocynin, an inhibitor of $\mathrm{NAD}(\mathrm{P}) \mathrm{H}$ oxidase, can delay cerebral vasospasm in a rat subarachnoid hemorrhage model [52], and it also has protective effects on pups with intraventricular hemorrhage [53]. However, a neuroprotective effect was not observed in a rat $\mathrm{ICH}$ model [54]. Perhaps the amount of ROS derived from $\mathrm{NAD}(\mathrm{P}) \mathrm{H}$ oxidase is small compared with the total amount of ROS produced in ICH such that the effect of apocynin is limited. Sulfaphenazole (SPZ), which can inhibit superoxide production by cytochrome $\mathrm{P} 450$, is also a ROS scavenger.
Hama et al. reported that systemic SPZ treatment reduces striatal dysfunction, elevated lipid peroxidation, and brain edema in a rat ICH model [55]. Thrombin can also initiate potentially harmful pathways, such as apoptosis in cultured neurons and astrocytes [56], and can activate Src kinase $[57,58]$, which may contribute to excitotoxicity and ROS release [59]. Therefore inhibition of thrombin may reduce injury induced by intracerebral hemorrhage. Mitochondria play an important role in ROS release in $\mathrm{ICH}$, as mentioned in Section 3. Ma et al. reported that the mitochondrial ROS scavenger Mito-TEMPO can decrease the amount of the ROS in ICH and that TRO-19622, an inhibitor of the MPTP, can also reduce RIRR [24].

Agents that scavenge free radicals constitute the second group. These compounds include melatonin (5-methoxy$N$-acetyl-tryptamine) and its metabolites, which are able to ameliorate early brain injury [60-62]. Melatonin has been shown to inhibit ROS related to red blood cell lysis and hemoglobin degradation. In addition, it can increase the expression of Nrf2-ARE pathway-related free radical scavengers, such as SOD and GSH, by activating the Nrf2ARE pathway [63]. Hydrogen, the lightest element in the periodic table and the most abundant chemical substance in the universe, also can be used as an effective antioxidant therapy. Ohsawa et al. reported that inhaled hydrogen gas has antioxidant and antiapoptotic properties that protect the brain against ischemia-reperfusion (I/R) injury and stroke by selectively reducing the hydroxyl radical [64]. In the ICH model, inhalation of hydrogen gas can reduce brain edema acutely $(24 \mathrm{~h})$, but it is not effective over a longer time frame (72 h) [65]. Momordica charantia polysaccharide (MCP), obtained from Momordica charantia, can also be an antioxidant agent. Duan et al. also have demonstrated that MCP scavenged ROS in intracerebral hemorrhage damage, attenuating neuronal death in primary hippocampal neurons [66]. Pyrroloquinoline quinone (PQQ), which has been proven to exist in various fruits, vegetables, milk, and even mammalian tissues, can antagonize oxidative stressinduced cell damage [67-69]. Lu et al. have reported that PQQ reduced the production of reactive oxygen species, alleviated brain edema, and improved locomotor function after ICH [70]. Redox nanoparticles may become a new type of treatment for ROS. These agents can solve the problems of traditional agents, including nonspecific dispersion in normal issues, preferential renal clearance, poor permeability across the $\mathrm{BBB}$, and rapid reduction to the corresponding hydroxylamine form. Chonpathompikunlert et al. reported that, in rats treated with redox polymer self-assembled nanoparticles (nitroxide radical-containing nanoparticles [RNPs]), significantly lower levels of superoxide anion and 8-OHdG (biomarkers of oxidative stress) were detected compared with rats in the control group. RNPs also ameliorated intracerebral hemorrhage-induced brain edema and neurological deficits [71].

There are many studies on antioxidant strategies in ICH, but there have been only a few drugs tested in clinical trials. Edaravone, a free radical scavenger, can significantly improve the outcome of ischemic stroke, as evaluated by the modified Rankin Scale, within 3 months and was introduced in Japan 
for clinical use in June 2001 [72]. Its neuroprotective effect was also proven in preclinical trials [73-75]. However, in a meta-analysis of edaravone for acute intracerebral hemorrhage, which included 10 randomized controlled trials (RCTs) involving 768 participants, although edaravone treatment increased the rate of improvement of neurological impairment within the scheduled treatment, it is not clear that this translated to any longer-term benefit of clinical importance, and the quality of each trial was not high [76]. Therefore, the longer-term benefit of edaravone is still unclear. NXY059, a free radical-trapping neuroprotectant, is also a clinically tested antioxidant. However, in a randomized control trial including 607 patients, there were no differences in 3-month function, disability, or neurological deficit scores between the experimental group and the control group [77].

\section{Conclusions}

Reactive oxygen species are increasingly recognized as important players in the pathophysiology of secondary brain damage after ICH. Mitochondria play an important part in the production of ROS and are necessary to the processes of neuronal cell death and BBB injury. Much effort has been put into the development of antioxidants to neutralize oxidative stress. We have investigated many intracellular and mitochondrial targets, aiming to decrease ROS release or eliminate released ROS in ICH. However, we know that nearly all attempts failed. There may be two reasons for this. ROS in cells are not always harmful; they also play a role in molecular signal transduction. Therefore, treatment should be aimed at cytotoxic radicals. Additionally, we should pay more attention to the time window in which drugs are administered because it is not easy to supply patients with drugs in time. Moreover, the local and systemic influences of reactive oxygen species in $\mathrm{ICH}$ patients remain to be better characterized.

\section{Competing Interests}

The authors declare that they have no competing interests.

\section{Authors' Contributions}

Jie Qu and Weixiang Chen contributed equally.

\section{Acknowledgments}

This study was funded by the National Basic Research Program of China (973 Program, 2014CB541600) and the National Natural Science Foundation of China (81501002, 81371340, and 81220108009).

\section{References}

[1] C. J. van Asch, M. J. Luitse, G. J. Rinkel, I. van der Tweel, A. Algra, and C. J. Klijn, "Incidence, case fatality, and functional outcome of intracerebral haemorrhage over time, according to age, sex, and ethnic origin: a systematic review and metaanalysis," The Lancet Neurology, vol. 9, no. 2, pp. 167-176, 2010.
[2] V. L. Feigin, C. M. Lawes, D. A. Bennett, S. L. Barker-Collo, and V. Parag, "Worldwide stroke incidence and early case fatality reported in 56 population-based studies: a systematic review," The Lancet Neurology, vol. 8, no. 4, pp. 355-369, 2009.

[3] R. F. Keep, Y. Hua, and G. Xi, "Intracerebral haemorrhage: mechanisms of injury and therapeutic targets," The Lancet Neurology, vol. 11, no. 8, pp. 720-731, 2012.

[4] P. D. Ray, B.-W. Huang, and Y. Tsuji, "Reactive oxygen species (ROS) homeostasis and redox regulation in cellular signaling," Cellular Signalling, vol. 24, no. 5, pp. 981-990, 2012.

[5] K. J. Davies, "Oxidative stress: the paradox of aerobic life," Biochemical Society Symposium, vol. 61, pp. 1-31, 1995.

[6] N. Han, S.-J. Ding, T. Wu, and Y.-L. Zhu, "Correlation of free radical level and apoptosis after intracerebral hemorrhage in rats," Neuroscience Bulletin, vol. 24, no. 6, pp. 351-358, 2008.

[7] M. Katsu, K. Niizuma, H. Yoshioka, N. Okami, H. Sakata, and P. H. Chan, "Hemoglobin-induced oxidative stress contributes to matrix metalloproteinase activation and blood-brain barrier dysfunction in vivo," Journal of Cerebral Blood Flow \& Metabolism, vol. 30, no. 12, pp. 1939-1950, 2010.

[8] D. B. Zorov, M. Juhaszova, and S. J. Sollott, "Mitochondrial reactive oxygen species (ROS) and ROS-induced ROS release," Physiological Reviews, vol. 94, no. 3, pp. 909-950, 2014.

[9] W. Dröge, "Free radicals in the physiological control of cell function," Physiological Reviews, vol. 82, no. 1, pp. 47-95, 2002.

[10] M. P. Murphy, "How mitochondria produce reactive oxygen species," Biochemical Journal, vol. 417, no. 1, pp. 1-13, 2009.

[11] S. R. Robinson, T. N. Dang, R. Dringen, and G. M. Bishop, "Hemin toxicity: a preventable source of brain damage following hemorrhagic stroke," Redox Report, vol. 14, no. 6, pp. 228 235, 2009.

[12] X.-Y. Xiong, J. Wang, Z.-M. Qian, and Q.-W. Yang, "Iron and intracerebral hemorrhage: from mechanism to translation," Translational Stroke Research, vol. 5, no. 4, pp. 429-441, 2014.

[13] Q. Chen, J. Tang, L. Tan et al., "Intracerebral hematoma contributes to hydrocephalus after intraventricular hemorrhage via aggravating iron accumulation," Stroke, vol. 46, no. 10, pp. 2902-2908, 2015.

[14] H. Wu, T. Wu, X. Xu, J. Wang, and J. Wang, "Iron toxicity in mice with collagenase-induced intracerebral hemorrhage," Journal of Cerebral Blood Flow \& Metabolism, vol. 31, no. 5, pp. 1243-1250, 2011.

[15] R. F. Regan, M. Chen, Z. Li, X. Zhang, L. Benvenisti-Zarom, and J. Chen-Roetling, "Neurons lacking iron regulatory protein-2 are highly resistant to the toxicity of hemoglobin," Neurobiology of Disease, vol. 31, no. 2, pp. 242-249, 2008.

[16] J. F. Clark, M. Loftspring, W. L. Wurster et al., "Bilirubin oxidation products, oxidative stress, and intracerebral hemorrhage," Acta Neurochirurgica, Supplementum, vol. 105, pp. 7-12, 2008.

[17] F. Sharp, D. Z. Liu, X. Zhan, and B. P. Ander, "Intracerebral hemorrhage injury mechanisms: glutamate neurotoxicity, thrombin, and Src," Acta Neurochirurgica. Supplementum, vol. 105, pp. 4346, 2008.

[18] D. C. Joshi, B. P. Tewari, M. Singh, P. G. Joshi, and N. B. Joshi, "AMPA receptor activation causes preferential mitochondrial $\mathrm{Ca}^{2+}$ load and oxidative stress in motor neurons," Brain Research, vol. 1616, pp. 1-9, 2015.

[19] E. Mracsko and R. Veltkamp, "Neuroinflammation after intracerebral hemorrhage," Frontiers in Cellular Neuroscience, vol. 8, article 388, 2014. 
[20] J. Wang and S. E. Tsirka, "Tuftsin fragment 1-3 is beneficial when delivered after the induction of intracerebral hemorrhage," Stroke, vol. 36, no. 3, pp. 613-618, 2005.

[21] M. B. Hampton, A. J. Kettle, and C. C. Winterbourn, "Inside the neutrophil phagosome: oxidants, myeloperoxidase, and bacterial killing," Blood, vol. 92, no. 9, pp. 3007-3017, 1998.

[22] H. X. Nguyen, T. J. O’Barr, and A. J. Anderson, "Polymorphonuclear leukocytes promote neurotoxicity through release of matrix metalloproteinases, reactive oxygen species, and TNF- $\alpha$," Journal of Neurochemistry, vol. 102, no. 3, pp. 900-912, 2007.

[23] P. Bernardi, A. Rasola, M. Forte, and G. Lippe, "The mitochondrial permeability transition pore: channel formation by F-ATP synthase, integration in signal transduction, and role in pathophysiology," Physiological Reviews, vol. 95, no. 4, pp. 11111155, 2015.

[24] Q. Ma, S. Chen, Q. Hu, H. Feng, J. H. Zhang, and J. Tang, "NLRP3 inflammasome contributes to inflammation after intracerebral hemorrhage," Annals of Neurology, vol. 75, no. 2, pp. 209-219, 2014.

[25] X. Wang, T. Mori, T. Sumii, and E. H. Lo, "Hemoglobin-induced cytotoxicity in rat cerebral cortical neurons: caspase activation and oxidative stress," Stroke, vol. 33, no. 7, pp. 1882-1888, 2002.

[26] L. Goldstein, Z.-P. Teng, E. Zeserson, M. Patel, and R. F. Regan, "Hemin induces an iron-dependent, oxidative injury to human neuron-like cells," Journal of Neuroscience Research, vol. 73, no. 1, pp. 113-121, 2003.

[27] S. J. Dixon, K. M. Lemberg, M. R. Lamprecht et al., "Ferroptosis: an iron-dependent form of nonapoptotic cell death," Cell, vol. 149, no. 5, pp. 1060-1072, 2012.

[28] P. Chang, W. Dong, M. Zhang et al., "Anti-necroptosis chemical necrostatin-1 can also suppress apoptotic and autophagic pathway to exert neuroprotective effect in mice intracerebral hemorrhage model," Journal of Molecular Neuroscience, vol. 52, no. 2, pp. 242-249, 2014.

[29] A. Degterev, Z. Huang, M. Boyce et al., "Chemical inhibitor of nonapoptotic cell death with therapeutic potential for ischemic brain injury," Nature Chemical Biology, vol. 1, no. 2, pp. 112-119, 2005.

[30] M. D. Laird, C. Wakade, C. H. Alleyne Jr., and K. M. Dhandapani, "Hemin-induced necroptosis involves glutathione depletion in mouse astrocytes," Free Radical Biology and Medicine, vol. 45, no. 8, pp. 1103-1114, 2008.

[31] P. Ballabh, A. Braun, and M. Nedergaard, "The blood-brain barrier: an overview: structure, regulation, and clinical implications," Neurobiology of Disease, vol. 16, no. 1, pp. 1-13, 2004.

[32] J. J. Chang, B. A. Emanuel, W. J. Mack, G. Tsivgoulis, and A. V. Alexandrov, "Matrix metalloproteinase-9: dual role and temporal profile in intracerebral hemorrhage," Journal of Stroke and Cerebrovascular Diseases, vol. 23, no. 10, pp. 2498-2505, 2014.

[33] B. M. Hybertson, B. Gao, S. K. Bose, and J. M. McCord, "Oxidative stress in health and disease: the therapeutic potential of Nrf2 activation," Molecular Aspects of Medicine, vol. 32, no. 46, pp. 234-246, 2011.

[34] X. Zhao, G. Sun, S.-M. Ting et al., "Cleaning up after ICH: the role of Nrf2 in modulating microglia function and hematoma clearance," Journal of Neurochemistry, vol. 133, no. 1, pp. 144152, 2015.

[35] J. Wang, J. Fields, C. Zhao et al., "Role of Nrf2 in protection against intracerebral hemorrhage injury in mice," Free Radical Biology and Medicine, vol. 43, no. 3, pp. 408-414, 2007.
[36] H. Shang, D. Yang, W. Zhang et al., "Time course of Keap1-Nrf2 pathway expression after experimental intracerebral haemorrhage: correlation with brain oedema and neurological deficit," Free Radical Research, vol. 47, no. 5, pp. 368-375, 2013.

[37] C. E. Guerrero-Beltrán, M. Calderón-Oliver, J. PedrazaChaverri, and Y. I. Chirino, "Protective effect of sulforaphane against oxidative stress: recent advances," Experimental and Toxicologic Pathology, vol. 64, no. 5, pp. 503-508, 2012.

[38] C. F. Chang, S. Cho, and J. Wang, "(-)-Epicatechin protects hemorrhagic brain via synergistic Nrf2 pathways," Annals of Clinical and Translational Neurology, vol. 1, no. 4, pp. 258-271, 2014.

[39] S. Sukumari-Ramesh and C. H. Alleyne, "Post-injury administration of tert-butylhydroquinone attenuates acute neurological injury after intracerebral hemorrhage in mice," Journal of Molecular Neuroscience, vol. 58, no. 4, pp. 525-531, 2016.

[40] X.-R. Zhao, N. Gonzales, and J. Aronowski, "Pleiotropic role of PPAR $\gamma$ in intracerebral hemorrhage: an intricate system involving Nrf2, RXR, and NF- $\kappa \mathrm{B}$," CNS Neuroscience and Therapeutics, vol. 21, no. 4, pp. 357-366, 2015.

[41] E. Y. Park, I. J. Cho, and S. G. Kim, "Transactivation of the PPAR-responsive enhancer module in chemopreventive glutathione S-transferase gene by the peroxisome proliferatoractivated receptor- $\gamma$ and retinoid X receptor heterodimer," Cancer Research, vol. 64, no. 10, pp. 3701-3713, 2004.

[42] S. C. Thal, M. Heinemann, C. Luh, D. Pieter, C. Werner, and K. Engelhard, "Pioglitazone reduces secondary brain damage after experimental brain trauma by PPAR- $\gamma$-independent mechanisms," Journal of Neurotrauma, vol. 28, no. 6, pp. 983-993, 2011.

[43] J.-H. Yi, S.-W. Park, N. Brooks, B. T. Lang, and R. Vemuganti, "PPAR $\gamma$ agonist rosiglitazone is neuroprotective after traumatic brain injury via anti-inflammatory and anti-oxidative mechanisms," Brain Research, vol. 1244, pp. 164-172, 2008.

[44] A. Cherubini, C. Ruggiero, M. C. Polidori, and P. Mecocci, "Potential markers of oxidative stress in stroke," Free Radical Biology and Medicine, vol. 39, no. 7, pp. 841-852, 2005.

[45] Y. C. Chen, C. M. Chen, J. L. Liu, S. T. Chen, M. L. Cheng, and D. T. Chiu, "Oxidative markers in spontaneous intracerebral hemorrhage: leukocyte 8 -hydroxy- $2^{\prime}$-deoxyguanosine as an independent predictor of the 30-day outcome," Journal of Neurosurgery, vol. 115, no. 6, pp. 1184-1190, 2011.

[46] C. J. Weir, S. W. Muir, M. R. Walters, and K. R. Lees, "Serum urate as an independent predictor of poor outcome and future vascular events after acute stroke," Stroke, vol. 34, no. 8, pp. 19511956, 2003.

[47] H. Wu, Q. Jia, G. Liu et al., "Decreased uric acid levels correlate with poor outcomes in acute ischemic stroke patients, but not in cerebral hemorrhage patients," Journal of Stroke and Cerebrovascular Diseases, vol. 23, no. 3, pp. 469-475, 2014.

[48] M. C. Polidori, P. Mecocci, and B. Frei, "Plasma vitamin C levels are decreased and correlated with brain damage in patients with intracranial hemorrhage or head trauma," Stroke, vol. 32, no. 4, pp. 898-902, 2001.

[49] Y. Hua, R. F. Keep, J. T. Hoff, and G. Xi, "Deferoxamine therapy for intracerebral hemorrhage," in Cerebral Hemorrhage, L. F. Zhou, X. C. Chen, and F. P. Huang, Eds., vol. 105 of Acta Neurochirurgica Supplement, pp. 3-6, 2008.

[50] G. Wang, W. Hu, Q. Tang et al., "Effect comparison of both iron chelators on outcomes, iron deposit, and iron transporters after intracerebral hemorrhage in rats," Molecular Neurobiology, 2015.

[51] T. Masuda, H. Hida, Y. Kanda et al., "Oral administration of metal chelator ameliorates motor dysfunction after a small 
hemorrhage near the internal capsule in rat," Journal of Neuroscience Research, vol. 85, no. 1, pp. 213-222, 2007.

[52] "Retraction. Inhibition of NADPH oxidase attenuates vasospasm after experimental subarachnoid hemorrhage in rats," Stroke, vol. 42, no. 7, p. 2117, 2011.

[53] M. T. Zia, A. Csiszar, N. Labinskyy et al., "Oxidative-nitrosative stress in a rabbit pup model of germinal matrix hemorrhage: role of NAD(P)H oxidase," Stroke, vol. 40, no. 6, pp. 2191-2198, 2009.

[54] E. Titova, R. P. Ostrowski, L. C. Sowers, J. H. Zhang, and J. Tang, "Effects of apocynin and ethanol on intracerebral haemorrhageinduced brain injury in rats," Clinical and Experimental Pharmacology and Physiology, vol. 34, no. 9, pp. 845-850, 2007.

[55] S. Hama, Y. Ishihara, M. Watanabe, S. Danjo, Y. Nakamura, and K. Itoh, "Effects of sulfaphenazole after collagenase-induced experimental intracerebral hemorrhage in rats," Biological and Pharmaceutical Bulletin, vol. 35, no. 10, pp. 1849-1853, 2012.

[56] F. M. Donovan, C. J. Pike, C. W. Cotman, and D. D. Cunningham, "Thrombin induces apoptosis in cultured neurons and astrocytes via a pathway requiring tyrosine kinase and RhoA activities," The Journal of Neuroscience, vol. 17, no. 14, pp. 53165326, 1997.

[57] D.-Z. Liu, B. P. Ander, H. Xu et al., "Blood-brain barrier breakdown and repair by Src after thrombin-induced injury," Annals of Neurology, vol. 67, no. 4, pp. 526-533, 2010.

[58] T. D. Ardizzone, X. Zhan, B. P. Ander, and F. R. Sharp, "Src kinase inhibition improves acute outcomes after experimental intracerebral hemorrhage," Stroke, vol. 38, no. 5, pp. 1621-1625, 2007.

[59] D.-Z. Liu, X.-Y. Cheng, B. P. Ander et al., "Src kinase inhibition decreases thrombin-induced injury and cell cycle re-entry in striatal neurons," Neurobiology of Disease, vol. 30, no. 2, pp. 201211, 2008.

[60] Y. Ueda, T. Masuda, A. Ishida et al., "Enhanced electrical responsiveness in the cerebral cortex with oral melatonin administration after a small hemorrhage near the internal capsule in rats," Journal of Neuroscience Research, vol. 92, no. 11, pp. 1499-1508, 2014.

[61] R. E. Hartman, H. A. Rojas, T. Lekic et al., "Long-term effects of melatonin after intracerebral hemorrhage in rats," in Cerebral Hemorrhage, vol. 105 of Acta Neurochirurgica Supplementum, pp. 99-100, Springer, Berlin, Germany, 2008.

[62] T. Lekic, R. Hartman, H. Rojas et al., "Protective effect of melatonin upon neuropathology, striatal function, and memory ability after intracerebral hemorrhage in rats," Journal of Neurotrauma, vol. 27, no. 3, pp. 627-637, 2010.

[63] Z. Wang, C. Ma, C.-J. Meng et al., "Melatonin activates the Nrf2ARE pathway when it protects against early brain injury in a subarachnoid hemorrhage model," Journal of Pineal Research, vol. 53, no. 2, pp. 129-137, 2012.

[64] I. Ohsawa, M. Ishikawa, K. Takahashi et al., "Hydrogen acts as a therapeutic antioxidant by selectively reducing cytotoxic oxygen radicals," Nature Medicine, vol. 13, no. 6, pp. 688-694, 2007.

[65] A. Manaenko, T. Lekic, Q. Ma, J. H. Zhang, and J. Tang, "Hydrogen inhalation ameliorated mast cell-mediated brain injury after intracerebral hemorrhage in mice," Critical Care Medicine, vol. 41, no. 5, pp. 1266-1275, 2013.

[66] Z.-Z. Duan, X.-L. Zhou, Y.-H. Li, F. Zhang, F.-Y. Li, and Q. Su-Hua, "Protection of Momordica charantia polysaccharide against intracerebral hemorrhage-induced brain injury through
JNK3 signaling pathway," Journal of Receptors and Signal Transduction, vol. 35, no. 6, pp. 523-529, 2015.

[67] K. Ohwada, H. Takeda, M. Yamazaki et al., "Pyrroloquinoline quinone (PQQ) prevents cognitive deficit caused by oxidative stress in rats," Journal of Clinical Biochemistry and Nutrition, vol. 42, no. 1, pp. 29-34, 2008.

[68] A. K. Singh, S. K. Pandey, and G. Naresh Kumar, "Pyrroloquinoline quinone-secreting probiotic escherichia coli nissle 1917 ameliorates ethanol-induced oxidative damage and hyperlipidemia in rats," Alcoholism: Clinical and Experimental Research, vol. 38, no. 7, pp. 2127-2137, 2014.

[69] R. Tao, J. S. Karliner, U. Simonis et al., "Pyrroloquinoline quinone preserves mitochondrial function and prevents oxidative injury in adult rat cardiac myocytes," Biochemical and Biophysical Research Communications, vol. 363, no. 2, pp. 257262, 2007.

[70] H. Lu, J. Shen, X. Song et al., "Protective effect of pyrroloquinoline quinone (PQQ) in rat model of intracerebral hemorrhage," Cellular and Molecular Neurobiology, vol. 35, no. 7, pp. 921-930, 2015.

[71] P. Chonpathompikunlert, C.-H. Fan, Y. Ozaki, T. Yoshitomi, C.-K. Yeh, and Y. Nagasaki, "Redox nanoparticle treatment protects against neurological deficit in focused ultrasoundinduced intracerebral hemorrhage," Nanomedicine, vol. 7, no. 7, pp. 1029-1043, 2012.

[72] E. Otomo, H. Tohgi, K. Kogure et al., "Effect of a novel free radical scavenger, edaravone (MCI-186), on acute brain infarction. Randomized, placebo-controlled, double-blind study at multicenters," Cerebrovascular Diseases, vol. 15, no. 3, pp. 222229, 2003.

[73] H. Shang, D. Cui, D. Yang, S. Liang, W. Zhang, and W. Zhao, "The radical scavenger edaravone improves neurologic function and perihematomal glucose metabolism after acute intracerebral hemorrhage," Journal of Stroke and Cerebrovascular Diseases, vol. 24, no. 1, pp. 215-222, 2015.

[74] T. Nakamura, Y. Kuroda, S. Yamashita et al., "Edaravone attenuates brain edema and neurologic deficits in a rat model of acute intracerebral hemorrhage," Stroke, vol. 39, no. 2, pp. $463-$ 469, 2008.

[75] F. Zhao and Z. Liu, "Beneficial effects of edaravone on the expression of serum matrix metalloproteinase- 9 after cerebral hemorrhage," Neurosciences, vol. 19, no. 2, pp. 106-110, 2014.

[76] J. Yang, M. Liu, J. Zhou, S. Zhang, S. Lin, and H. Zhao, "Edaravone for acute intracerebral haemorrhage," Cochrane Database of Systematic Reviews, no. 2, Article ID CD007755, 2011.

[77] P. D. Lyden, A. Shuaib, K. R. Lees et al., "Safety and tolerability of NXY-059 for acute intracerebral hemorrhage: the CHANT trial," Stroke, vol. 38, no. 8, pp. 2262-2269, 2007. 


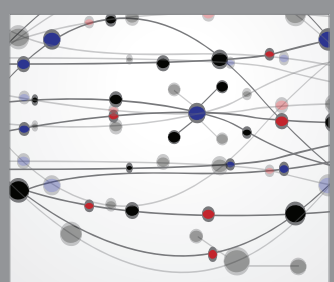

The Scientific World Journal
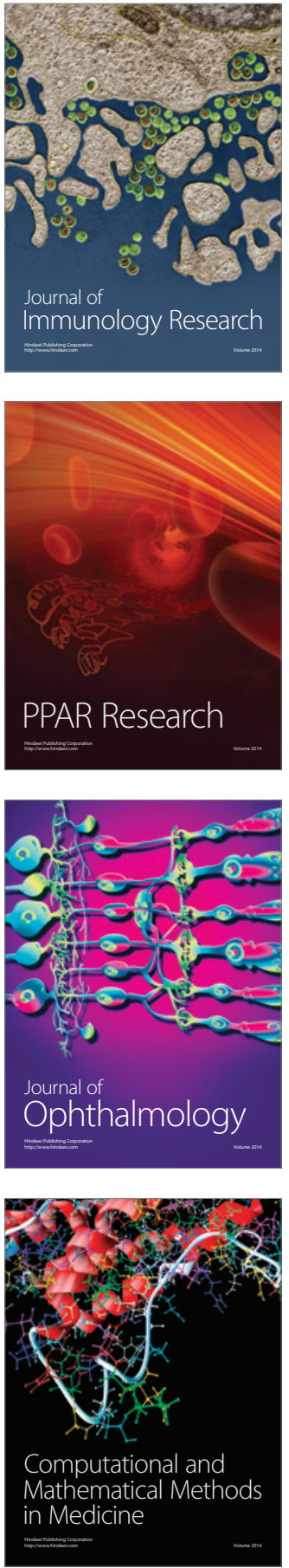

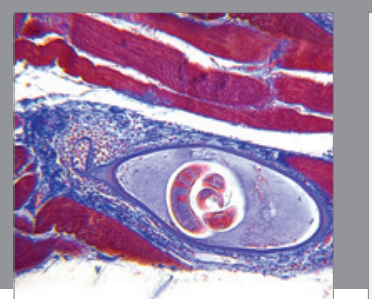

Gastroenterology Research and Practice

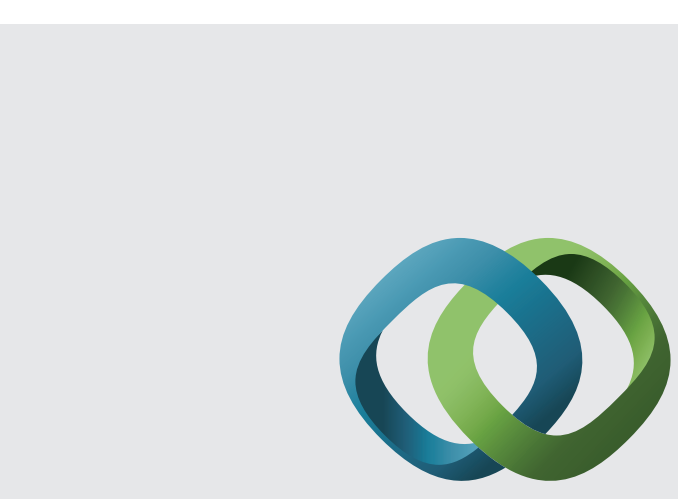

\section{Hindawi}

Submit your manuscripts at

http://www.hindawi.com
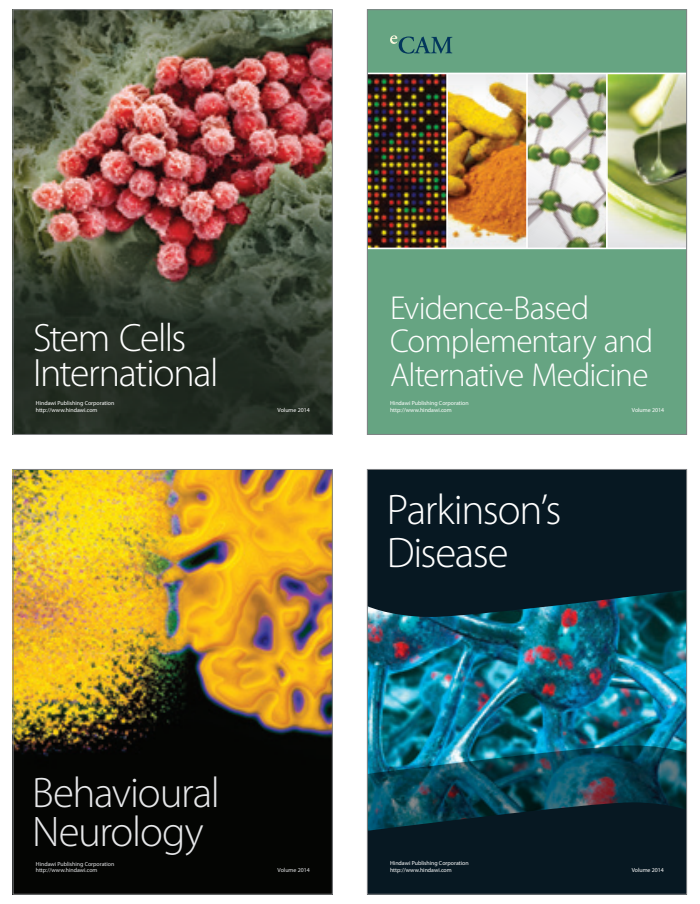
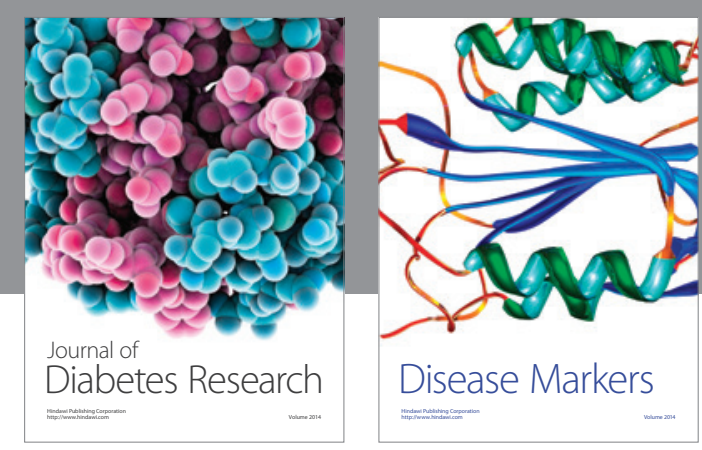

Disease Markers
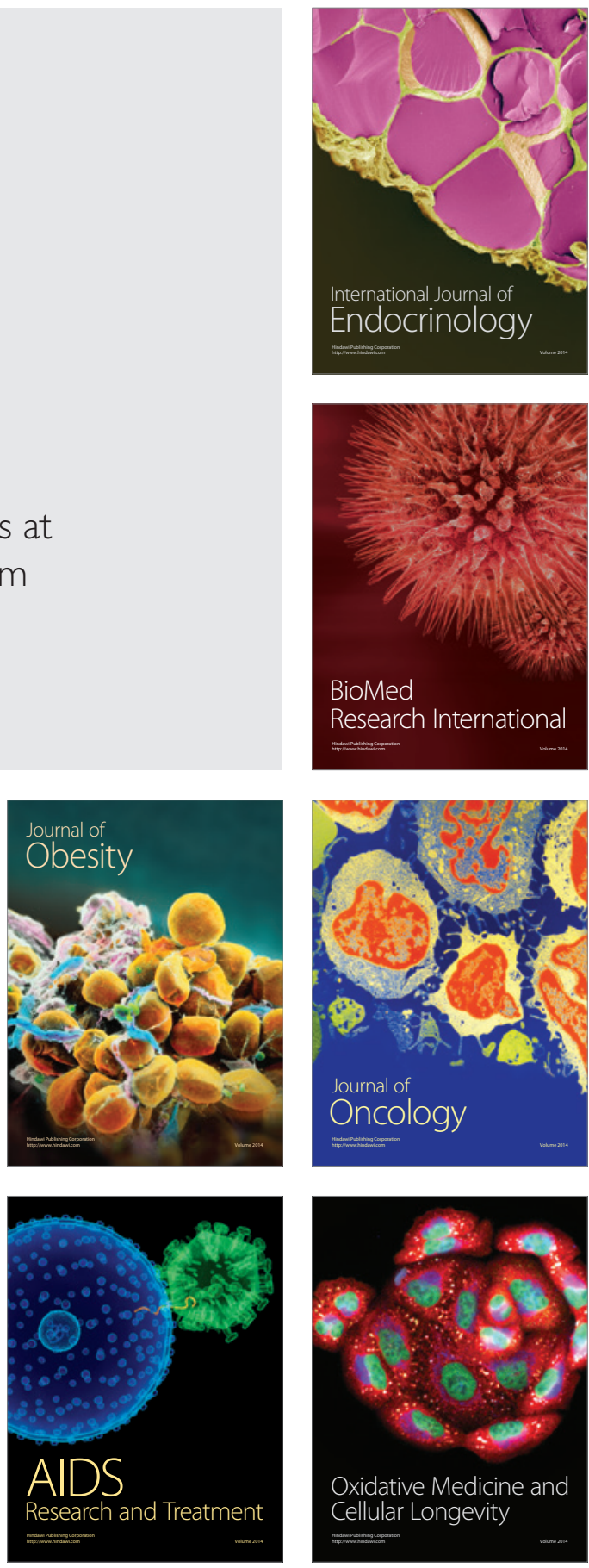